\title{
Relationship Between Attachment Site of Tibialis Anterior Muscle and Shape of Tibia: Anatomical Study of Cadavers
}

\section{Kentaro Kimata}

Aichi Medical University: Aichi lka Daigaku https://orcid.org/0000-0002-3773-0619

Shun Otsuka ( $\nabla$ tsukashun0512@gmail.com)

Aichi Medical University School of Medicine https://orcid.org/0000-0003-1832-9472

Hiroki Yokota

Meijo University: Meijo Daigaku

Xiyao Shan

Aichi Medical University: Aichi Ika Daigaku

Naoyuki Hatayama

Aichi Medical University: Aichi Ika Daigaku

Munekazu Naito

Aichi Medical University: Aichi Ika Daigaku

Research

Keywords: tibialis anterior muscle, tibia, S-like curve, cadaver, sex differences

Posted Date: March 7th, 2022

DOI: https://doi.org/10.21203/rs.3.rs-1379773/v1

License: (9) (i) This work is licensed under a Creative Commons Attribution 4.0 International License.

Read Full License 


\section{Abstract}

Background: Tibialis anterior (TA) muscle is the largest dorsiflexor of the ankle joint and plays an important role during gait movement. However, descriptions of the TA attachment site are inconsistent even among major anatomy textbooks, and its origin, especially the attachment site for the tibia, has not been reported in detail. This study is the first experimental attempt to investigate the origin of the TA in detail, paying particular attention to the relationship with the shape of the tibia, including sex differences.

Methods: Forty legs (20 males, 20 females) from twenty Japanese cadavers were examined. Gross anatomical examination of the TA's attachment site to the tibia and the tibia's shape was performed.

Results: The location of the distal end of the TA's attachment on tibia was more distal in males than in females. The anterior border of the tibia had a gentle S-like curve, with a medially convex curve in the proximal region and a laterally convex curve in the distal region in frontal plane. The most protruding point of the distal curve of the anterior border locates more proximal in females than in males.

Conclusions: There were sex differences in relationship between the distal end of the attachment site on tibia of the TA and the shape of the tibia. Consequently, in males, the TA was considered to be advantageous for power exertion, while in females it worked efficiently for ankle movements. Sex differences in TA's attachment site and the shape of the tibia may be involved in gait movement as well as frequency of lower leg disorders such as chronic exertional compartment syndrome.

\section{Introduction}

The tibialis anterior (TA) muscle is the largest muscle in the anterior compartment of the lower leg, accounting for over $60 \%$ of the ankle dorsiflexor muscle volume [1, 2]. The TA contributes to the inversion and dorsiflexion of the ankle joint and is involved in maintaining the medial arch of the foot [3]. During locomotion, the TA is active during the heel strike and swing phases to control foot drop and prevent tripping, respectively $[4,5]$. Loading of the TA is suggested as a determining factor of the walk-to-run transition speed $[4,6]$. The TA is one of the most important muscles in daily life because it is deeply involved in enabling erect bipedalism.

Several studies have examined the morphological properties of the TA. Wolf and Kim [7] reported that the TA is a pennate muscle composed of three partitions: superficial longitudinal fibers, deep longitudinal fibers, and oblique fibers. The fibers of the TA tendon reportedly rotate approximately 90 degrees from the musculotendinous junction to their insertion on the medial cuneiform and first metatarsal bone [8,9]. Furthermore, the tendon of the TA has individual variations, such as the number of bands, thicknesses, and insertion sites $[9,10]$. On the other hand, several major anatomy textbooks have described that the TA originates from $1 / 2$ to $2 / 3$ of the distance proximal to the lateral surface of the tibia, which is inconsistent with each other [11-13]. Individual differences were also observed in the length of the TA muscle belly. 
Several studies have compared gait movement between men and women and reported that stride length was significantly longer in men, while cadence was significantly greater in women [14-16]. These studies imply that the morphological variations of the TA affect ankle dorsiflexion efficiency and the sex differences in gait. It is also important to elucidate the origin of the TA to understand the motor characteristics of the ankle joint during walking motion. However, the anatomical features of the TA that might be different between sexes in gait have not been examined. The anterior border of the tibia is an important attachment site of the TA and appears to have a gentle S-like curve. As the TA runs along the tibia, this S-like curve influences the attachment pattern and function of the TA. To the best of our knowledge, the origin of the TA, especially the attachment site for the tibia, has not been reported in detail. This study is the first to investigate the origin of the TA in detail for the first time, paying particular attention to the relationship with the shape of the tibia, including sex differences.

\section{Materials And Methods}

Twenty Japanese cadavers (10 male, 10 female) were examined in this study. The ethics committee of Aichi Medical University School of Medicine approved this study (approval no. 2020-M131). The mean \pm standard deviation age of the cadavers was $83.2 \pm 8.0$ years (males, $82.3 \pm 5.7$ years; females, $84.1 \pm 9.7$ years). Forty legs ( 20 males, 20 females) were dissected to assess the attachment site of the TA to the tibia; none showed any apparent signs of deformation or atrophy.

Skin, adipose, and connective tissues were manually removed from the cadavers' legs. Gross anatomical examination of the TA's attachment site to the tibia and the tibia's shape was performed. The TA tendon was cut at the ankle joint level and carefully turned laterally to determine the origin of the tibia. The site where muscle fibers firmly adhered to the bone cortex was defined as the origin of the TA (Figs. 1-a, 1-b). Then, the distal ends of the tibial attachment of the anterior and posterior fibers of the TA were identified (Figs. 1-c, 1-d). The anterior and posterior fibers of the TA were defined as the fibers located anterior and posterior to the central aponeurosis itself, respectively. An S-curve of the anterior border of the tibia, with medially convex curve in the proximal region and laterally convex curve in the distal region in frontal plane, was also observed (Fig. 1-b). The lower legs were set to maximize the widths of the medial and lateral condyles and the following parameters were measured in 1-mm increments: tibial length (distance between the upper end of the medial condyle and the top of the medial malleolus) and the distances between the upper end of the medial condyle and points A, P, S1, and S2 (distance A, P, S1, and S2, respectively). All distances were quantified by dividing by the individual tibial length $(T)$ and are shown as percentages (A-T ratio, P-T ratio, S1-T ratio, and S2-T ratio, respectively) (Fig. 2).

\section{Statistical analysis}

Pearson product-moment correlation was used to investigate the relationships between tibial length and the distances A, P, S1, and S2. Sex and right-left differences in the tibial attachment site of the TA and the shape of the tibia were examined using an independent sample $t$-test and a paired $t$-test, respectively. $\mathrm{R}$ 
4.0.2 (R Foundation for Statistical Computing, Vienna, Austria) was used for all the statistical analyses. The significance level was set at $p<0.05$.

\section{Results}

The anterior fibers of the TA originated from the lateral condyle and anterior border of the tibia, while the posterior fibers originated from the anterolateral surface of the tibia and the anterior surface of the interosseous membrane. The TA transitioned to a flat tendon while descending the anterolateral surface of the tibia, passed under the extensor retinaculum at the anterior lower end of the lower leg, and then inserted into the medial cuneiform and the first metatarsal bone. The TA was composed of anterior and posterior fibers according to their positional relationship with respect to the central aponeurosis (Fig. 3). The anterior border of the tibia descended from the lateral side of the tibial tuberosity toward the medial malleolus, showing a gentle S-curve. It included a medial convex curve in the proximal region and a lateral convex curve in the distal region of the tibia.

The anatomical measurements of the TA and tibia are presented in Table 1. In the observed specimens, the tibial length was significantly longer in male $(329 \pm 16 \mathrm{~mm})$ than in female $(315 \pm 18 \mathrm{~mm})$ cadavers $(p=0.01$ ). Distance A was $152 \pm 18 \mathrm{~mm}$ (males, $160 \pm 14 \mathrm{~mm}$; females, $143 \pm 18 \mathrm{~mm}, p<0.01$ ). There was a positive correlation between distance $A$ and tibial length in males $(r=0.70, p<0.001)$ versus no correlation in females (Fig. 4). The A-T ratio was significantly larger in males $(48.7 \pm 3.2 \%)$ than in females $(45.5 \pm 5.3 \%)(p=0.03)$ (Fig. 5). Distance P was slightly longer in males $(216 \pm 23 \mathrm{~mm})$ than in females $(210 \pm 24 \mathrm{~mm})$. The mean P-T ratio was $66.1 \pm 6.4 \%$ and showed no significant sex differences (males: $65.6 \pm 6.7 \%$, females: $66.7 \pm 6.4 \%)$. The mean distance $S 1$ of males $(111 \pm 10 \mathrm{~mm}$ ) was longer than that of females $(103 \pm 8 \mathrm{~mm})(p<0.01)$. There was a positive correlation between distance $\mathrm{S} 1$ and tibial length in both males $(r=0.53, p=0.02)$ and females $(r=0.45, p<0.05)$ (Fig. 4). The S1-T ratio was not significantly different between males $(33.7 \pm 2.7 \%)$ and females $(32.7 \pm 2.4 \%)$. Distance $S 2$ was longer in males $(201 \pm 15 \mathrm{~mm})$ than in females $(185 \pm 15 \mathrm{~mm})(p<0.01)$, while a positive correlation was observed between distance S2 and tibial length in both males $(r=0.68, p<0.01)$ and females $(r=0.80, p<$ $0.001)$ (Fig. 4). The S2-T ratio was significantly smaller in females $(58.7 \% \pm 2.9 \%)$ than in males $(61.2 \pm$ $3.4 \%)(p=0.02)$ (Fig. 5). No significant differences in all parameters were detected between right and left sides. 
Table 1

Anatomical measurements of the tibia and points $A, P, S 1$, and S2

\begin{tabular}{|c|c|c|c|c|}
\hline & $\begin{array}{l}\text { Total } \\
(n=40)\end{array}$ & $\begin{array}{l}\text { Male } \\
(n=20)\end{array}$ & $\begin{array}{l}\text { Female } \\
(n=20)\end{array}$ & $\begin{array}{l}\text { p-value } \\
\text { (Male vs Female) }\end{array}$ \\
\hline Tibial length (mm) & $322 \pm 18$ & $329 \pm 16$ & $315 \pm 18$ & 0.01 \\
\hline Distance A (mm) & $152 \pm 18$ & $160 \pm 14$ & $143 \pm 18$ & $<0.01$ \\
\hline Distance P (mm) & $213 \pm 23$ & $216 \pm 23$ & $210 \pm 24$ & 0.48 \\
\hline Distance S1 (mm) & $107 \pm 10$ & $111 \pm 10$ & $103 \pm 8$ & $<0.01$ \\
\hline Distance S2 (mm) & $193 \pm 17$ & $201 \pm 15$ & $185 \pm 15$ & $<0.01$ \\
\hline A-T ratio $(\%)$ & $47.1 \pm 4.6$ & $48.7 \pm 3.2$ & $45.5 \pm 5.3$ & 0.03 \\
\hline P-T ratio (\%) & $66.2 \pm 6.4$ & $65.6 \pm 6.7$ & $66.7 \pm 6.4$ & 0.57 \\
\hline S1-T ratio (\%) & $33.2 \pm 2.6$ & $33.7 \pm 2.7$ & $32.7 \pm 2.4$ & 0.20 \\
\hline S2-T ratio (\%) & $59.9 \pm 3.3$ & $61.2 \pm 3.4$ & $58.7 \pm 2.9$ & 0.02 \\
\hline
\end{tabular}

\section{Discussion}

This study focused on the detailed information of the TA's attachment site to the tibia in addition to the previous studies that have examined the morphology of the TA. This study revealed sex differences in the relative position of the TA's attachment site on the tibia.

The TA was attached to the different levels of the tibia as the distal end of the anterior and posterior fibers (Fig. 1). In this study, the distal ends of the tibial attachment of the anterior fibers were located more proximally than the posterior fibers. According to the major anatomical textbook, the TA arises from the lateral condyle, proximal half to two-thirds of the lateral surface of the tibial shaft, deep surface of the deep fascia, and intermuscular septum between itself and the extensor digitorum longus [13]. The present study showed that the anterior and posterior fibers of the TA were located at $47.1 \pm 4.6 \%$ and $66.1 \pm 6.4 \%$ of the proximal tibia, respectively. The description of "proximal half" in the textbooks corresponds to the distal ends of the attachment of anterior fiber, while that of "two-thirds" corresponds to that of the posterior fiber.

The location of the distal end of the TA's attachment was more distal in males than in females. Assuming that the length of the tibia and the total length of the TA were constant, extending the muscle attachment area distally increased in the proportion of the muscle belly. Manal et al. investigated the pennation angle of the lower limb muscles using ultrasonography [17]. According to this, male subjects had significantly 
larger pennation angles for the TA at both rest and maximum voluntary contraction compared with the females. The larger pennation angle in males could be explained by the fact that the TA's attachment site extended more distal. These findings are considered to be beneficial for force exertion. Conversely, the anterior fibers of females have relatively longer tendon components than those of males. The longer tendon component and smaller pennation angle of the TA are considered more advantageous for efficient ankle dorsiflexion. According to previous studies, males and females walked at nearly the same mean preferred speed, while males had longer strides and females had higher cadences [16]. Therefore, during dorsiflexion of the ankle joint, the TA of females may work more efficiently to increase cadence or stride length than that in males according to their morphologies.

Chronic exertional compartment syndrome (CECS) is a common cause of lower leg pain in recreational and competitive athletes $[18,19]$. This syndrome is defined as reversible ischemia within a closed fibroosseous space, which leads to decreased tissue perfusion and ischemic pain [18]. Although there are several reports describing the incidence of CECS, the gender distribution has not been inconsistent yet [19-25]. A large retrospective cohort study of 1867 individuals suspected of having CECS was recently conducted [19]. According to this study, a larger proportion of men were diagnosed with CECS, and multivariable analysis confirmed that male sex was associated with a higher likelihood of a CECS diagnosis. However, these studies did not anatomically examine sex differences in the affected part. Since CECS is mainly caused by increased internal pressure in the anterior compartment of the lower leg, the anterior fibers of the TA extending more distally in males than in females is considered to be one of the risk factors of CECS.

The anterior border of the tibia had a gentle S-curve, with a medially convex curve in the proximal region and a laterally convex curve in the distal region. This is thought to provide a wide area for the TA's origin in the proximal region and play the role of a pulley that regulates the sliding direction of the tendon in the distal portion. In this study, the most protruding point of the distal curve of the anterior border (S2) was located more proximal in females than in males, whereas the most protruding point of the proximal curve (S1) was not different between sexes. The distal end of the tibia is more laterally rotated (tibial torsion) than the proximal end [13]. Furthermore, tibial torsion progresses throughout childhood and adolescence to the point of skeletal maturity, reaching 20-30 degrees in adults [26-28]. It is presumed that the distal part of the S-curve of the anterior border runs medially due to tibial torsion. It was also reported that tibial torsion is significantly greater in females than in males [27]. Females have relatively shorter leg lengths but greater tibial torsion than males. This may be the reason female cadavers presented a smaller S2-T ratio than the male cadavers. The proximal S2 distance makes the tendon of the TA closer to vertical and may make ankle dorsiflexion more efficient, especially in females.

\section{Limitation}

Although this study has clarified the relationship between attachment site of the TA and shape of tibia for the first time, it has some limitations. In this study, we did not analyze the strength of the TA and the 
range of motion of the ankle, because the specimens were from formalin-fixed cadavers. To discuss the dorsiflexion of the ankle, we would like to acquire and analyze these data in the future.

\section{Conclusion}

This study investigated the attachment site of the TA and the shape of the tibia. This revealed that the anterior and posterior fibers of the TA originated from the $1 / 2$ and $2 / 3$ of the distance proximal to the lateral surface of the tibia, respectively. There were sex differences in the relationship between the attachment site of the TA and the shape of the tibia. The TA was considered to be advantageous for force exertion in males, whereas to work efficiently for ankle movements in females.

\section{Abbreviations}

TA: tibialis anterior muscle, CECS: chronic exertional compartment syndrome

\section{Declarations}

\section{Acknowledgements}

The authors wish to express their gratitude to all those who donated their bodies to medical science. They thank Daisuke Mizuno and Takanori Kusumoto for their contributions to this study. They would like to thank Editage(www.editage.jp) for English language editing.

\section{Ethics approval and consent to participate}

This study has been approved by the ethics committee of Aichi Medical University School of Medicine (approval no. 2020-M131). The donors signed documents confirming their agreement to donate their bodies for use in clinical studies before their death. The format of the document was in accordance with the expectation of the Japanese law "Act on Body Donation for Medical and Dental Education". There are no materials that require permission to reproduce from other sources.

\section{Consent for publication}

Not applicable.

\section{Availability of data and materials}

The dataset used and analyzed during the current study are available from the corresponding author on reasonable request.

\section{Competing interests}

The authors declare that they have no competing interests. 


\section{Funding}

This research received no specific grant from any funding agency in the public, commercial, or not-forprofit sectors.

\section{Authors' contributions}

$\mathrm{KK}, \mathrm{SO}, \mathrm{NH}$ and $\mathrm{MN}$ were involved in the conception and design of the study. $\mathrm{KK}$ and $\mathrm{SO}$ acquired the data. KK, SO, HY and XS analyzed and interpreted the data. KK, SO and XS drafted the manuscript which was reviewed by SO, XS, NH and MN. All authors read and approved the final manuscript.

\section{References}

1. Fukunaga T, Roy RR, Shellock FG, Hodgson JA, Day MK, Lee PL, et al. Physiological cross-sectional area of human leg muscles based on magnetic resonance imaging. J Orthop Res. 1992;10(6):92834.

2. Miller SC, Korff T, Waugh C, Fath F, Blazevich AJ. Tibialis anterior moment arm: effects of measurement errors and assumptions. Med Sci Sports Exerc. 2015;47(2):428-39.

3. Basmajian JV, Stecko G. The role of muscles in arch support of the foot. J Bone Joint Surg Am. 1963;45:1184-90.

4. Bartlett JL, Kram R. Changing the demand on specific muscle groups affects the walk-run transition speed. J Exp Biol. 2008;211:1281-8.

5. Byrne CA, O'Keeffe DT, Donnelly AE, Lyons GM. Effect of walking speed changes on tibialis anterior EMG during healthy gait for FES envelope design in drop foot correction. J Electromyogr Kinesiol. 2007;17(5):605-16.

6. Hreljac A, Imamura RT, Escamilla RF, Edwards WB, MacLeod T. The relationship between joint kinetic factors and the walk-run gait transition speed during human locomotion. J Appl Biomech. 2008;24(2):149-57.

7. Wolf SL, Kim JH. Morphological analysis of the human tibialis anterior and medial gastrocnemius muscles. Acta Anat. 1997;158(4):287-95.

8. Fennell CW, Phillips P, 3rd. Redefining the anatomy of the anterior tibialis tendon. Foot Ankle Int. 1994;15(7):396-9.

9. Olewnik $\measuredangle$, Podgórski M, Polguj $M$, Topol M. A cadaveric and sonographic study of the morphology of the tibialis anterior tendon - a proposal for a new classification. J Foot Ankle Res. 2019;12:9.

10. Willegger M, Seyidova N, Schuh R, Windhager R, Hirtler L. Anatomical Footprint of the Tibialis Anterior Tendon: Surgical Implications for Foot and Ankle Reconstructions. Biomed Res Int. 2017;2017:9542125.

11. Gilroy AM, MacPherson BR, Schünke M, Schulte E, Schumacher U, Voll M, et al. Atlas of anatomy. New York: Thieme; 2016. 
12. Netter FH. Atlas of human anatomy. Philadelphia: Elsevier; 2018.

13. Gray H SS, Anand N. Gray's Anatomy: the anatomical basis of clinical practice. London: Elsevier; 2016.

14. Ko SU, Tolea MI, Hausdorff JM, Ferrucci L. Sex-specific differences in gait patterns of healthy older adults: results from the Baltimore Longitudinal Study of Aging. J Biomech. 2011;44(10):1974-9.

15. Kerrigan DC, Todd MK, Della Croce U. Gender differences in joint biomechanics during walking: normative study in young adults. Am J Phys Med Rehabil. 1998;77(1):2-7.

16. Bruening DA, Frimenko RE, Goodyear CD, Bowden DR, Fullenkamp AM. Sex differences in whole body gait kinematics at preferred speeds. Gait Posture. 2015;41(2):540-5.

17. Manal K, Roberts DP, Buchanan TS. Optimal pennation angle of the primary ankle plantar and dorsiflexors: variations with sex, contraction intensity, and limb. J Appl Biomech. 2006;22(4):255-63.

18. George CA, Hutchinson MR. Chronic exertional compartment syndrome. Clin Sports Med. 2012;31(2):307-19.

19. de Bruijn JA, van Zantvoort APM, van Klaveren D, Winkes MB, van der Cruijsen-Raaijmakers $M$, Hoogeveen AR, et al. Factors predicting lower leg chronic exertional compartment syndrome in a large population. Int J Sports Med. 2018;39(1):58-66.

20. Davis DE, Raikin S, Garras DN, Vitanzo P, Labrador H, Espandar R. Characteristics of patients with chronic exertional compartment syndrome. Foot Ankle Int. 2013;34(10):1349-54.

21. de Fijter WM, Scheltinga MR, Luiting MG. Minimally invasive fasciotomy in chronic exertional compartment syndrome and fascial hernias of the anterior lower leg: short- and long-term results. Mil Med. 2006;171(5):399-403.

22. Detmer DE, Sharpe K, Sufit RL, Girdley FM. Chronic compartment syndrome: diagnosis, management, and outcomes. Am J Sports Med. 1985;13(3):162-70.

23. Pedowitz RA, Hargens AR, Mubarak SJ, Gershuni DH. Modified criteria for the objective diagnosis of chronic compartment syndrome of the leg. Am J Sports Med. 1990;18(1):35-40.

24. Qvarfordt P, Christenson JT, Eklöf B, Ohlin P, Saltin B. Intramuscular pressure, muscle blood flow, and skeletal muscle metabolism in chronic anterior tibial compartment syndrome. Clin Orthop Relat Res. 1983(179):284-90.

25. Waterman BR, Liu J, Newcomb R, Schoenfeld AJ, Orr JD, Belmont PJ, Jr. Risk factors for chronic exertional compartment syndrome in a physically active military population. Am J Sports Med. 2013;41(11):2545-9.

26. Kristiansen LP, Gunderson RB, Steen H, Reikerås O. The normal development of tibial torsion. Skeletal Radiol. 2001;30(9):519-22.

27. Yoshioka Y, Siu DW, Scudamore RA, Cooke TD. Tibial anatomy and functional axes. J Orthop Res. 1989;7(1):132-7.

28. Eckhoff DG, Kramer RC, Watkins JJ, Burke BJ, Alongi CA, Stamm ER, et al. Variation in tibial torsion. Clin Anat. 1994;7(2):76-9. 
Figures

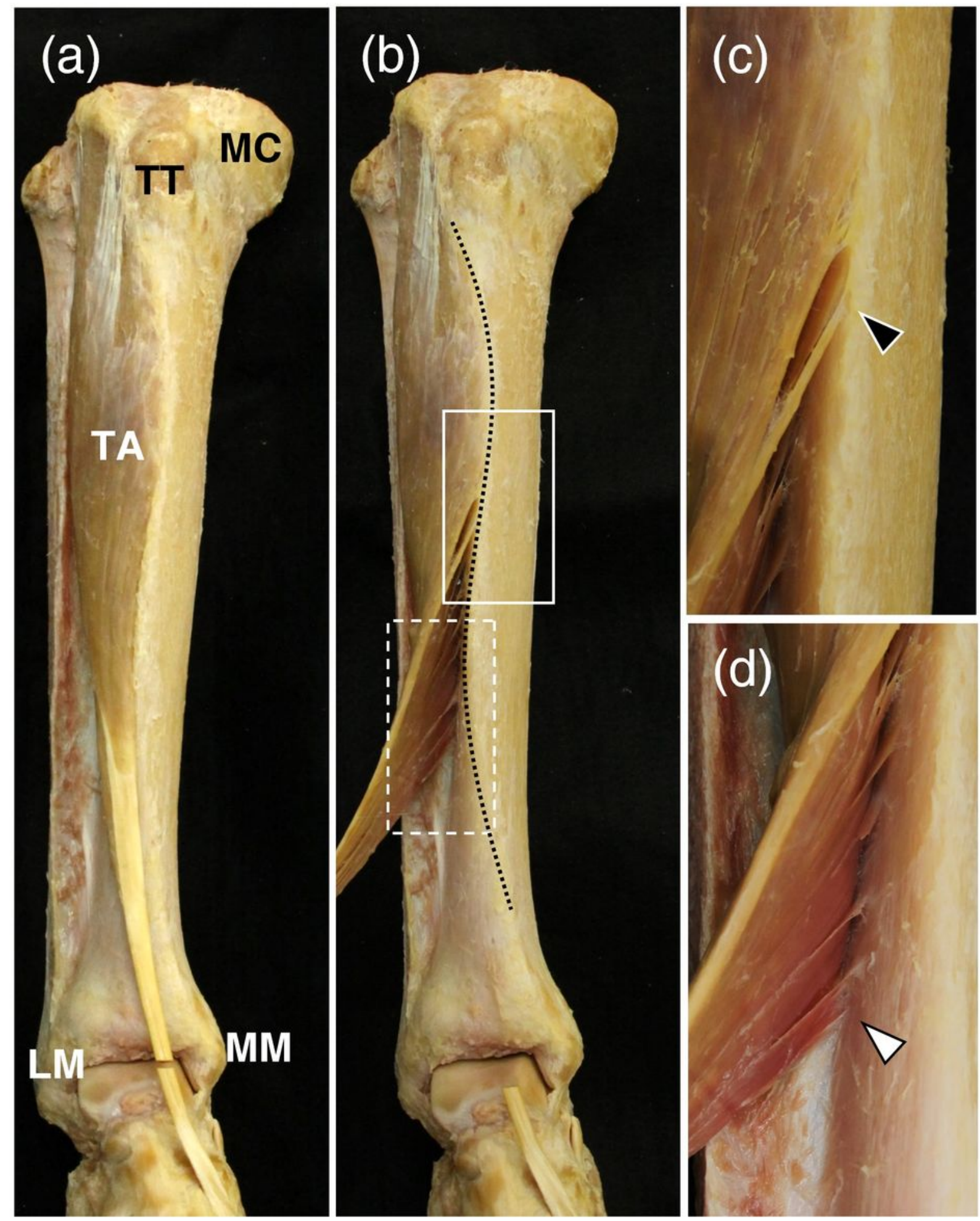

Figure 1

Dissection of the tibialis anterior muscle (TA) 
(a) Anterior view of the right TA (the tendon was cut at the ankle joint level). (b) The distal ends of the tibial attachment of the anterior and posterior fibers of the TA are shown. The distal part of the TA was turned over. The dotted curve shows the anterior border of the tibia. (c) and (d) Enlarged images of the white solid and dashed squares of (a), respectively. The black and white arrowheads indicate the most distal attachment points of the anterior and posterior fibers of the TA to the tibia. LM, lateral malleolus; $\mathrm{MC}$, medial condyle; MM, medial malleolus; $\mathrm{TA}$, tibialis anterior; $\mathrm{TT}$, tibial tuberosity

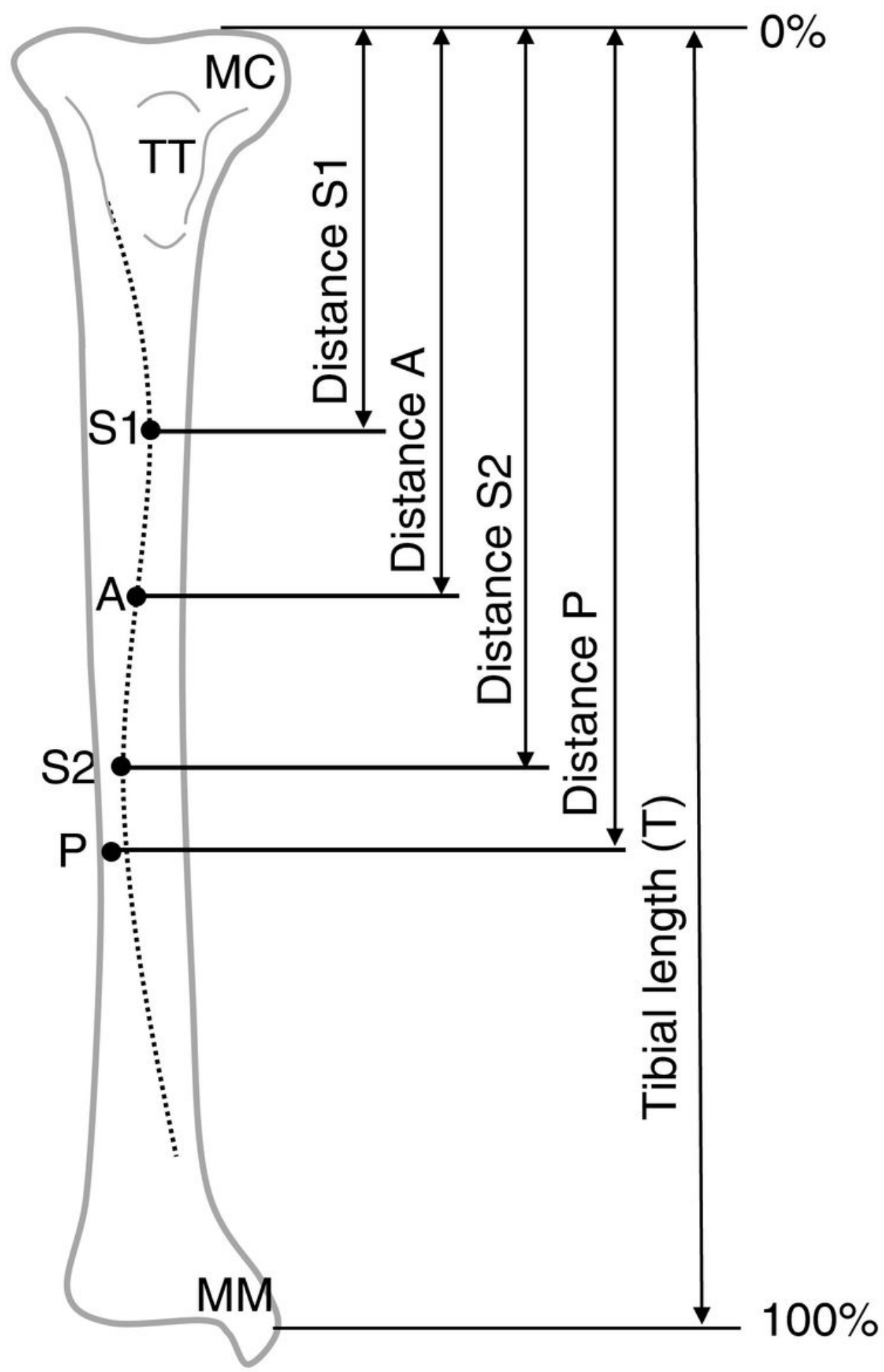




\section{Figure 2}

Measurement of the parameters of the TA and the tibia

The tibial length was measured from the upper end of the medial condyle to the top of the medial malleolus. Points $A$ and $P$ indicate the levels of attachment of the anterior and posterior fibers of the TA, respectively. The dotted S-curve shows the anterior border of the tibia. The levels at which the S-curve protrudes most medially and laterally are indicated as $S 1$ and $S 2$, respectively. The length from the upper end of the medial condyle to points A, P, S1, and S2 were measured (distances A, P, S1, and S2, respectively). The ratio of the length from the upper end of the medial condyle to points $A, P, S 1$, and $S 2$ to the tibial length $(T)$ was calculated as a percentage (A-T, P-T, S1-T, and S2-T ratios, respectively). MC, medial condyle; MM, medial malleolus; TT, tibial tuberosity
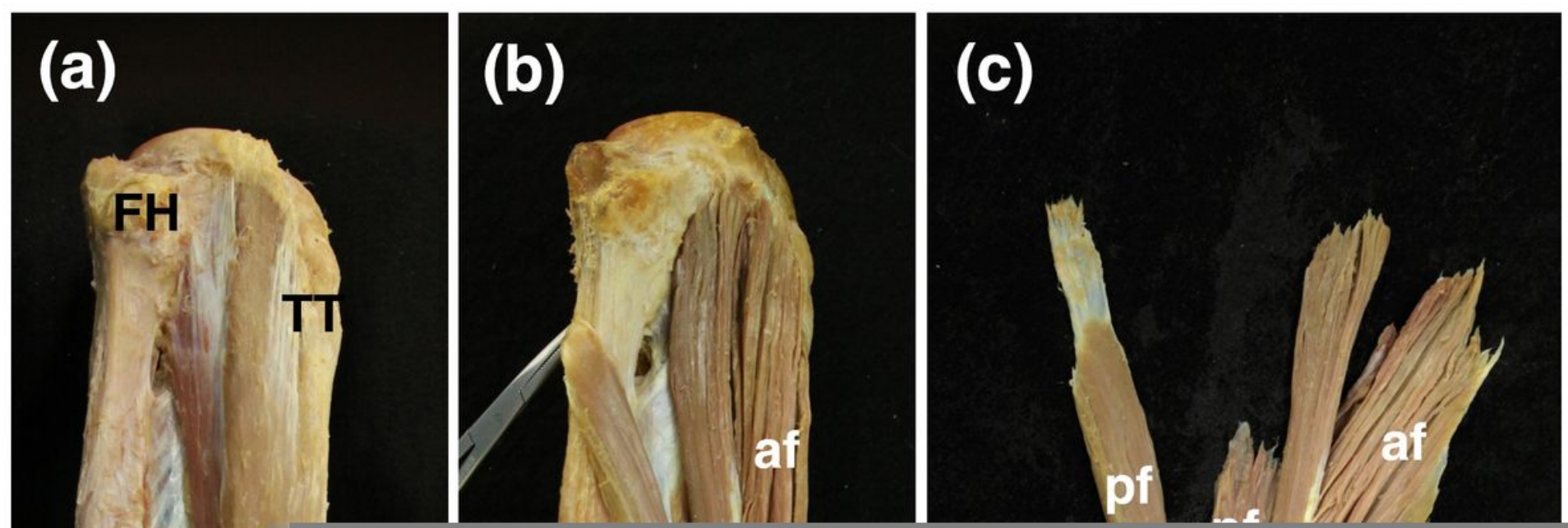
Figure 3

Anterior and posterior fibers of the TA

(a) Lateral view of the right leg. (b) The central aponeurosis was exposed and parts of the posterior fibers were flipped by forceps. The anterior and posterior fibers were defined by their positional relationship with the central aponeurosis. (c) The TA was removed from the tibia. The anterior and posterior fibers were reflected anteriorly and posteriorly to the central aponeurosis, respectively. af, anterior fiber; $\mathrm{FH}$, fibular head; pf, posterior fiber; TT, tibial tuberosity; asterisk, central aponeurosis

(a) Distance A

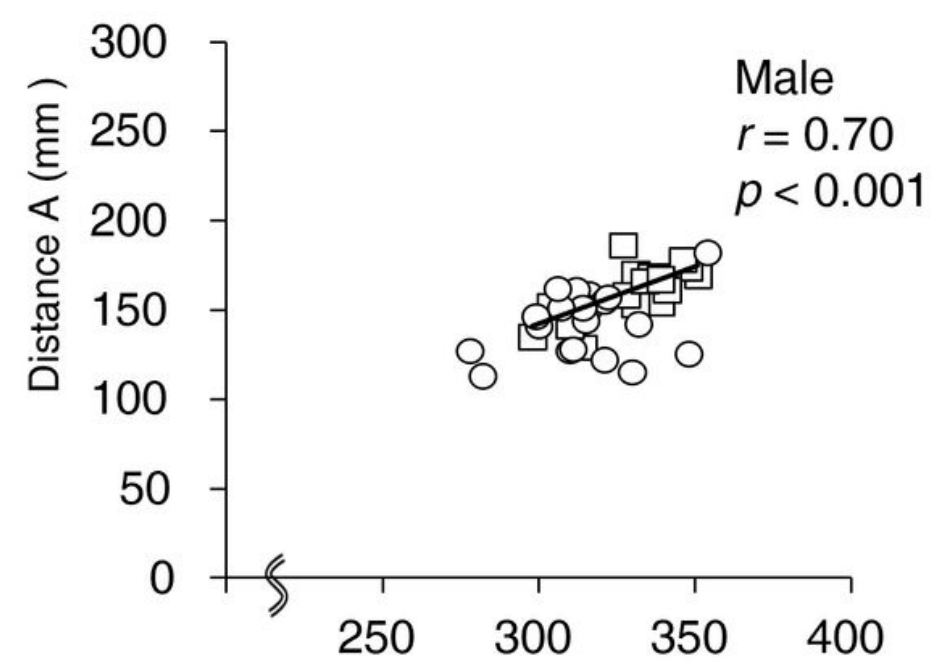

(c) Distance S1

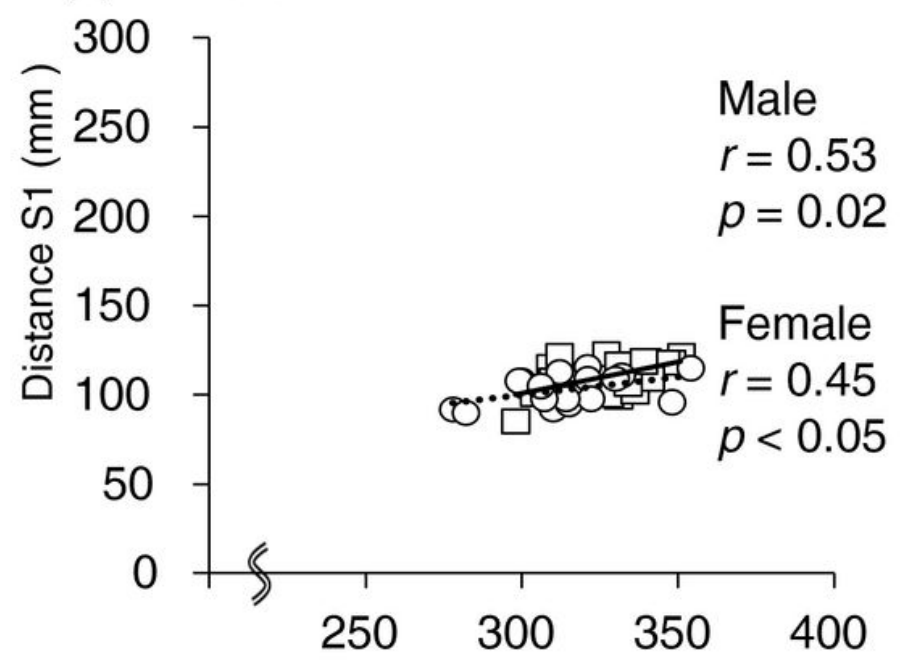

(b) Distance P

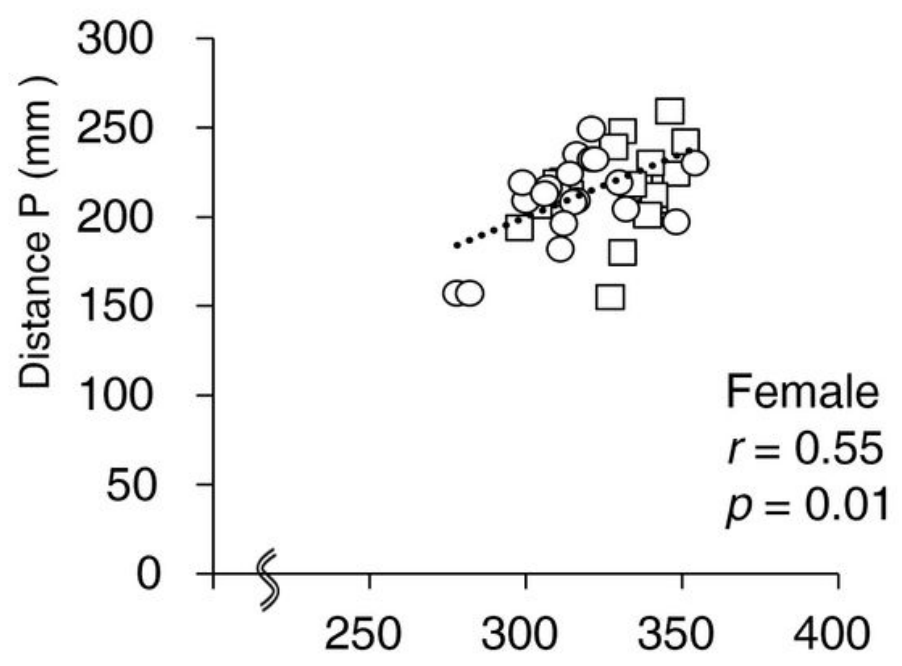

(d) Distance S2

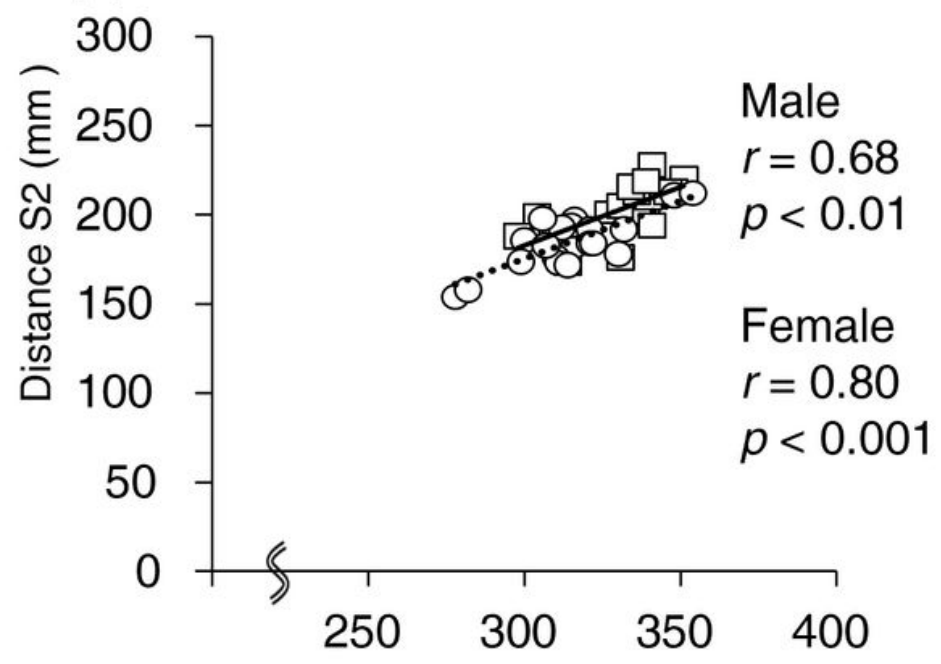

Tibial length ( $\mathrm{mm}$ )

Figure 4

Scatter plots between tibial length and distances A, P, S1, and S2 
All values are shown in millimeters. The solid and dotted lines show approximate lines of males and females, respectively. Distance A in females and distance $S 1$ in male were not correlated with tibial length. White squares, male; White circles, female

(a) A-T ratio

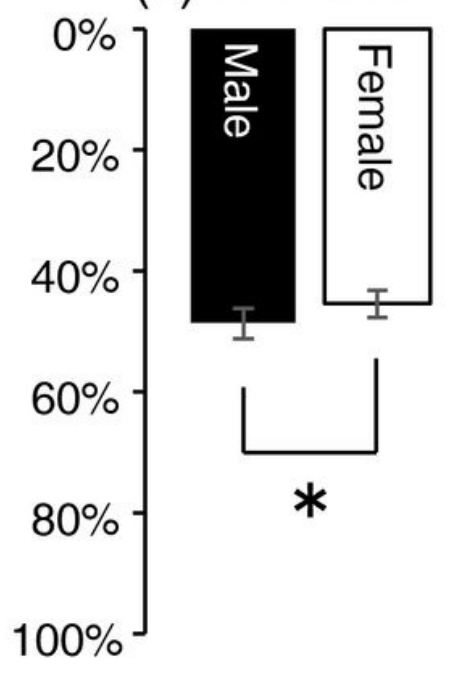

(b) P-T ratio

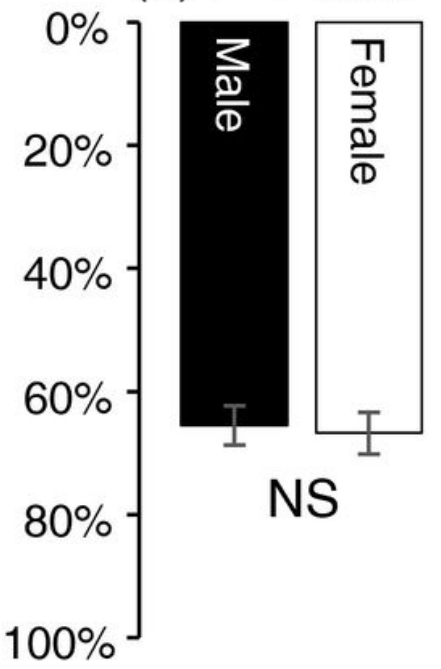

(c) S1-T ratio

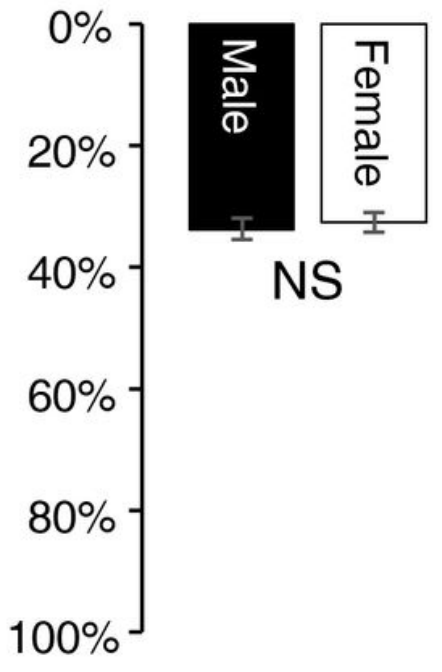

(d) S2-T ratio

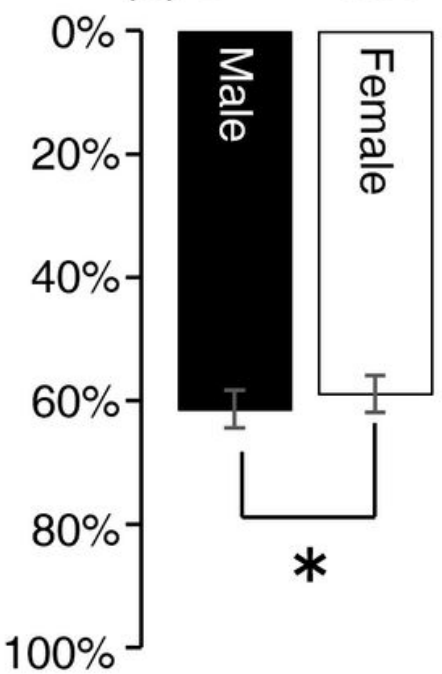

Figure 5

Statistical analysis of the tibialis anterior and tibia.

(a and b) A-T ratio was significantly larger in males than in females $(p=0.03)$, while P-T ratio did not differ significantly between males and females. (c and d) S1-T ratio did not differ significantly between the sexes, while the S2-T ratio was significantly larger in males than in females $(p=0.02)$. ${ }^{\star} p<0.05$; NS, not significant 